\title{
Present and future of sentinel lymph node biopsy in breast cancer staging
}

\author{
Antonio Piñero
}

$T^{\prime}$ here is little, if any, doubt about the feasibility and clinical applicability of sentinel lymph node biopsy (SLNB) in the management of breast cancer patients. Prognostic significance of lymph nodes is a major factor in the most used clinical and pathological staging system in breast cancer - the tumour, node, metastasis (TNM) classification system of the American Joint Committee on Cancer (AJCC) [1]. Since SLNB was described and began to be used in clinical practice in the early 1990s, many changes have been implemented from a technical point of view, especially those related to indications criteria.

Most criteria for SLNB not initially considered as good staging methods have been reconsidered, and we now find few absolute contraindications for the method. Changes in SLNB indications have defined three groups: initial indications that have not changed and are still absolute, initial absolute contraindications that have become indications or relative contraindications, and initial absolute contraindications that remain.

In the first group we find ductal and lobular invasive breast carcinomas $<3 \mathrm{~cm}$ in diameter (many authors increase this limit to $\mathrm{T} 2$, that is, $5 \mathrm{~cm}$ in diameter) and intraductal breast carcinoma $>2 \mathrm{~cm}$, high grade, or when mastectomy is needed. In this issue of Clinical and Translational Oncology, Collado et al. [2] confirm these selective criteria for SLNB indication in cases of intraductal tumours. They analyse 65 cases following these criteria and find that the use of SLNB allows unnecessary lymphadenectomy to be avoided in half of the patients that require mastectomy, and they conclude that indication for SLNB in patients with intraductal carcinoma of the breast should be proportional to the infiltration risk of the lesion.

The second group shows the highest number of changes. Previous cosmetic breast surgery, even with implants use, or

\footnotetext{
A. Piñero (局)

Department of General Surgery

Virgen de la Arrixaca University Hospital

Ctra. Madrid-Cartagena, s/n

ES-30120 El Palmar, Murcia, Spain

e-mail: antonio.pinero@carm.es
}

conservative surgery for breast cancer allows SLNB biopsy if it is necessary to stage a new tumour. This is supported by a dynamic concept of SLNB: sentinel node represents lymphatic drainage from a specific anatomic area and, if a previous surgical procedure has changed it, a new lymphatic net will develop in this basin with a "new representative" sentinel node. Breast cancer during pregnancy was also an initial contraindication for SLNB due to the use of radioactive tracers. Safety and feasibility studies have shown that foetal irradiation is not high enough to be considered harmful [3], so it has also become a potential application. Neoadjuvant or systemic primary therapy is a therapeutic modality that has increasingly been adopted in recent decades. Although it was only used to treated unresectable tumours, it is now applied in locally advanced tumours to try to avoid radical surgery and allow conservative techniques. This type of treatment was considered an absolute contraindication for SLNB, but many authors now agree that SLNB could be done in some of these patients at least $[4,5]$. It must be taken into account that neoadjuvant chemotherapy is used in a heterogeneous group of patients, and current problems are to define what patients can benefit (locally advanced cancers, node responders to neoadjuvant therapy) and the timing of SLNB (before or after neoadjuvant treatment).

The third group includes cases in which SLNB is not indicated. These are patients with inflammatory carcinoma of the breast and patients with obvious lymph node affectation. Even in some of these cases, some authors consider SLNB after systemic primary therapy administration.

Methodology is other important fact related to SLNB. Although indications have been extended and are better defined, the technical methodology continues to be heterogeneous, and there is no unique or specific protocol to follow. Almost each centre uses different tracers, different timing between tracer injection and surgery, different anatomical locations to inject the tracers, etc. Despite this, we can see similar results in identification and false negative rates, with few fixed differences such as finding more extraaxillary drainages in deeper injection of the tracer versus subareolar or subdermal injection.

We can therefore say that SLNB is a good diagnostic technique for breast cancer staging being indicated more 
and more frequently. However, what future innovations can we expect to improve its results? Surely these innovations will take place at three different fronts: first, we must consider new techniques and technological improvements. In this field, the development of new tracers, different size molecules, and mixed coloured isotopic tracer could show a higher and more specific intraoperative identification rate. For instance, the number of identified sentinel nodes depends on the molecule size: the bigger the molecule, the lower the number of scintigraphicdetected nodes. This happens with the time they are identified as well: the smaller the molecule, the faster the sentinel node shows up. Adjustment of these tracers' characteristics can help distinguish between real sentinel node and accessories nodes that, in this last case, should not be resected. In this respect, the use of intraoperative portable gamma cameras allows the visual identification of sentinel nodes and helps to distinguish between sentinel and accessory nodes by quantifying their level of radioactive activity [6].

Second, there will be innovations in the methods of analysing the isolated node or nodes. These will focus on finding the most accurate method of detecting tumour cells and, even possibly, intraoperatively. After being biopsied, the sentinel node can be studied using cytology, serial sections, staining with hematoxylin-eosin and immunohistochemical techniques. Currently a new molecular methodology is being applied: the one-step nucleic acid amplification technique (OSNA) by which expression of cytokeratin-19 RNA is detected in the sentinel node [7, 8].
There are important advantages associated with OSNA: It is an intraoperative test that shows higher sensibility and specificity than other cytological or histopathological intraoperative techniques. It studies the whole node, so postoperative analysis of this is unnecessary (and impossible). As a disadvantage, some authors argue that with this method, information about histopathology of the node cannot be attained and, for example, it is not possible to know if extranodal or capsular infiltration exists. In that respect, other question is the usefulness of such data in a node affected by macrometastases.

Lastly, in the coming years, there will be an integration of SLNB with other diagnostic tests. Diagnostic algorithms that make up several imaging techniques will be designed. The aim of such algorithms should be the addition of SLNB in the management of patients who could benefit from it and to avoid diagnosis delay due to unnecessary tests when they have not enough influence on therapeutic decisions. In this context, future studies using preoperative positron emission tomography (PET) and the spread of ultrasound-guided biopsies should make SLNB a more effective and efficient diagnostic tool.

In summary, SLNB is a key tool in the management of breast cancer, and as we have seen above, some important advances can improve its results in the future. Although many and important advances are being made, especially in the field of molecular biology, to find new predictive and prognostic parameters in breast cancer we can say that SLNB will continue being important while lymph node status is needed in breast cancer staging.

\section{References}

1. Edge SB, Byrd DR, Compton CC et al (eds) (2010) AJCC cancer staging manual, 7th edn. Springer-Verlag. New York

2. Collado MV, Ruiz-Tovar J, García-Villanueva A et al (2010) Sentinel lymph node biopsy in selected cases of ductal in situ breast carcinoma. Clin Transl Oncol 12:499-502

3. Gentilini O, Cremonesi M, Toesca A et al (2010)
Sentinel lymph node biopsy in pregnant patients with breast cancer. Eur J Nucl Med Mol Imaging 37:78-83

4. Straver ME, Rutgers EJTh, Russell NS et al (2009) Towards rational axillary treatment in relation to neoadjuvant therapy in breast cancer. Eur J Cancer 45:2284-2292.

5. Chung A, Giuliano A (2010) Axillary staging in the neoadjuvant setting. Ann Surg Oncol. Doi: 10.1245/s10434-010-1001-8

6. Mathelin C, Salvador S, Bekaert V et al (2008) A new intraoperative gamma camera for the sentinel lymph node procedure in breast cancer. Anticancer Res 28:2859-2864

7. Tamaki Y, Akiyama F, Iwase T et al (2009) Molecular detection of lymph node metastases in breast cancer patients: results of a multicenter trial using the one-step nucleic acid amplification assay. Clin Cancer Res 15:2879-2884

8. Bernet Vegué L, Martinez Benaclocha M, Cano Muñoz R et al (2010) Molecular diagnosis of sentinel lymph nodes for breast cancer: one step ahead for standardisation. Diag Mol Pathol (in press) 